\title{
HARDWARE SOFTWARE CO-DESIGN FOR A CLOSED LOOP CONTROL SYSTEM
}

\author{
B. Devi ${ }^{1}$, Sanju Prakash. $K^{2}$, Vijay Kumar. $S^{3}$ and Rohan P. Ganpati ${ }^{4}$
}

\begin{abstract}
The implementation of a hardware software co-design in the field of control systems is explained in this paper. The architecture of such systems is a key aspect in the design process. The system is designed based on the ATmega 328P microcontroller. The functionality of such a system is shown using a case study - Speed control of a DC motor under loaded and unloaded condition. Usage of minimal hardware and maximal software ensures reduction in hardware and lessens expenditure of the system and the dependency of the control system on the hardware is reduced. This makes the system design simplified and compacted. Purpose of speed control is to reduce the error and drive the motor at a set speed under loaded or unloaded condition to a certain extent. The speed control of DC motor is crucial in applications where precision is of essence.
\end{abstract}

Keywords - Arduino,DC Motor, PID Controller, Control system

\section{INTRODUCTION}

With growing technology pertaining to industrial automation, the demand for control systems have increased. Systems in which the output quantity has no effect upon the input to the control process is called an open-loop control system. The goal of any control system is to measure, monitor, and control a process. We can accurately control the process by monitoring its output and feeding some of it back, to compare the actual output with the desired output so as to reduce the error and bring the output of the system back to the desired response. The quantity of the output being measured is called the feedback signal, and the type of control system which uses feedback signals to both control and adjust itself is called a closed-loop system. Generally, systems modelled for these applications are mostly mechanical and require large size hardware. Some important applications are rolling mills, paper mills, printing presses, textile mills, excavators, cranes, mine winders, hoists, machine tools, traction. In embedded systems, the software and hardware must be designed together to make sure that the implementation not only functions properly but also meet the performance, cost and reliability goals. This paper uses a software based PID control system which reduces the hardware requirement and thereby reducing the overall cost of the system. PID (Proportional, Integral, and Derivative) control is a widely-used method to achieve and maintain a process set point. The process itself can vary widely, ranging from temperature control to speed control in miniature electric motors to position control of an inkjet printer head. While the applications vary widely, the approach in each case remains quite similar

\section{Problem Statement}

Today, control system applications are mostly mechanical and requires large size hardware. Mass scale production of these systems becomes expensive. Usage of minimal hardware and maximal software ensures reduction in hardware and as a positive outcome, cost and component size are reduced. This approach leads to increase in robustness and flexibility of the system. Conventionally used method is to implement PID hardware suitable for a specific application. This restricts the usage of the same hardware for various other applications. Thus, the need for a

${ }^{1}$ Assistant Professor, Electronics and Communication, Rajalakshmi Engineering College, Chennai, India

${ }^{2}$ Assistant Professor, Electronics and Communication, Rajalakshmi Engineering College, Chennai, India

${ }^{3}$ Assistant Professor, Electronics and Communication, Rajalakshmi Engineering College, Chennai, India

${ }^{4}$ Assistant Professor, Electronics and Communication, Rajalakshmi Engineering College, Chennai, India 
more flexible system has led to the software based approach for the PID controller. The emergence of hardware software co-design in the field of control systems has led to increase in customization and ever declining cost. A commonly used hardware in control system, a DC motor, is taken as a case study to elaborate this design approach of a low cost closed loop control system.

\section{MethodologY}

\section{A. System Design}

The system design comprises of an ATmega 328P microcontroller, 12V DC Supply, Voltage Regulator LM7812, Transistor n-p-n 2N2222, Diode, 12V DC Motor, IR Sensor, Potentiometer, 16x2 Liquid Crystal Display (LCD).

\section{B. System operation}

A $12 \mathrm{~V} \mathrm{DC}$ motor, for which the actual point is to be controlled based on the set point and load. Voltage regulator is used to maintain a steady voltage level in a circuit despite the fluctuations in the input. The output from the regulator is used to power the motor and the LCD. An LCD display is used to display the required input and output data for the system. An IR proximity sensor works by applying a voltage to IR light emitting diode which emit infrared light. This light hits the chopper of the motor and it is reflected back towards the sensor.

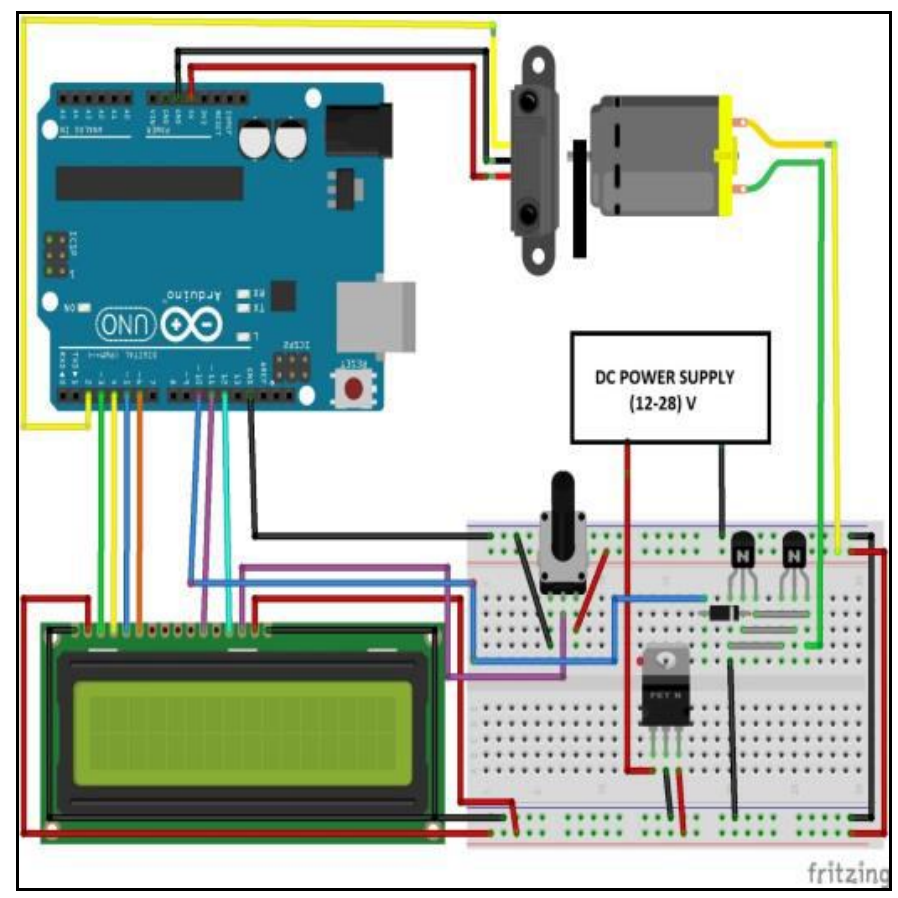

Figure 1. System schematic

The transistor is used as a switch that uses just a little current from the Arduino digital output to control the much bigger current of the motor. The diode connected across the connections of the motor only allows electricity to flow in one direction. When the power is turned off the circuit gets a negative spike of voltage that can damage your Arduino or the transistor. The diode protects against this by shorting out any such reverse current from the motor. 


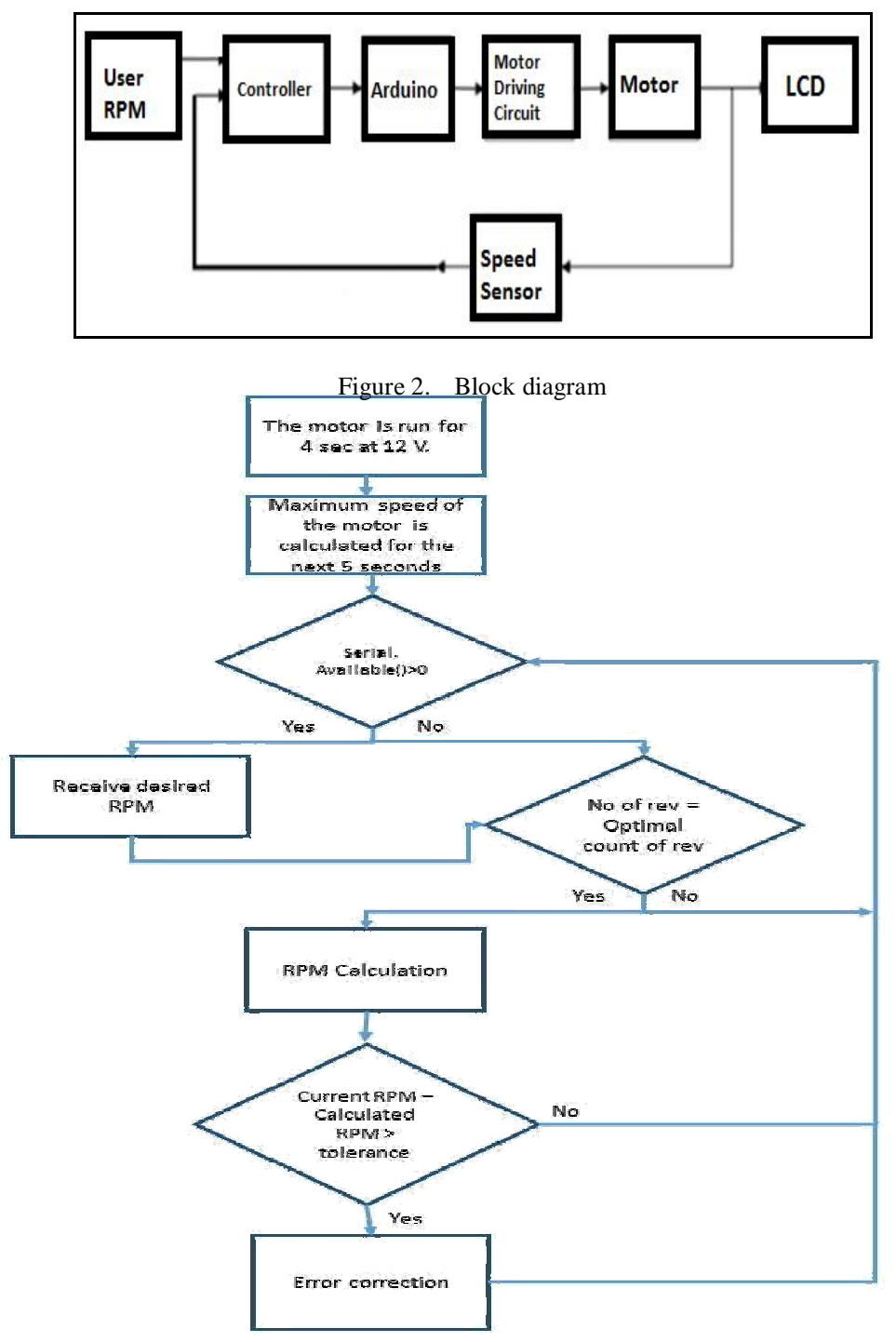

Figure 3. Flow diagram

The speed of the motor is calculated by noting the time taken by the motor to complete a certain optimal count of revolutions and computing with the equation: RPM $=60000 *$ optimal count of revolution /time taken. The difference between the actual point and the set point gives the error. This error is diminished by the PID technique, where a certain amount of correction is being done to the original PWM signal.

\section{Feedback}

The motor response is observed to be nonlinear and the PWM mapping using the direct proportional method doesn't make the motor run at the set point. This concludes that the motor doesn't have a linear behavior in the whole operating range. 


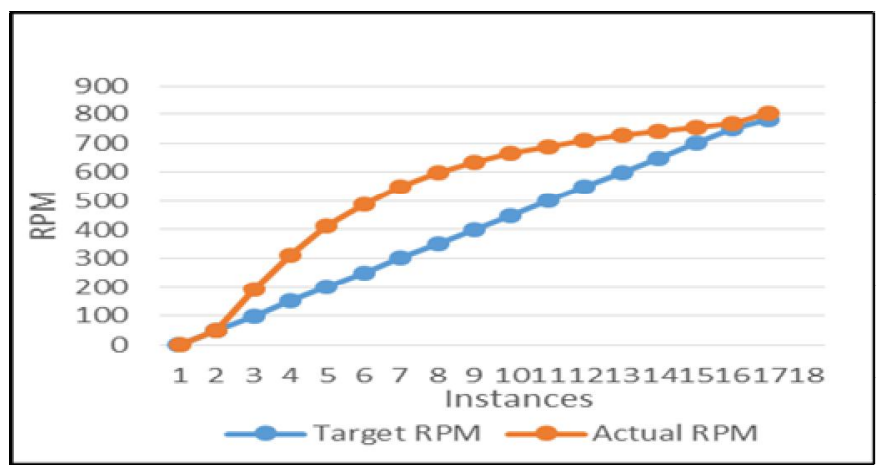

Figure 4. Motor response without feedback

The change in the speed of the motor when the user inputs a value is brought about by an algorithm which keeps checking the difference in the actual point and the set point and feeds a corrected PWM based on the value of the difference. This difference is calculated based on a PID control algorithm.

The Proportional-Integral-Derivative (PID) controller is a generic control loop feedback mechanism widely used in control systems. The PID control equation may be expressed as:

\section{Correction $=K \mathrm{~K}^{*}$ Error $+\mathrm{Ki} * \Sigma$ Error $+\mathrm{Kd} * \mathrm{dP} / \mathrm{dT}$}

Where, error is the difference between the set point and the actual point; $\Sigma$ error is the summation of previous error values; and $\mathrm{dP} / \mathrm{dT}$ is the time rate of change of speed.

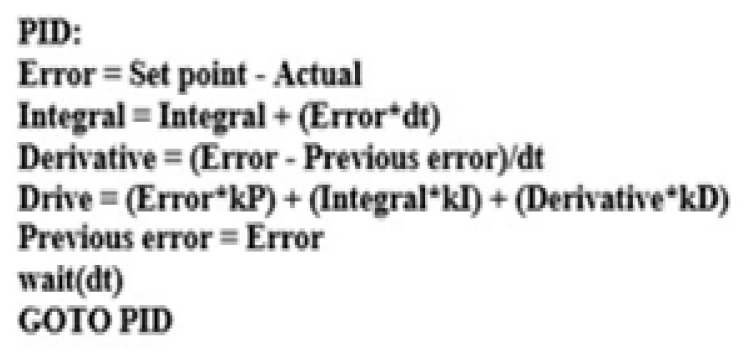

Figure 5. Pseudocode for PID

Proportional gain (Kp): Larger proportional gain typically means faster response, larger the error, larger is the proportional term compensation. However, an excessively large proportional gain results in process instability and oscillation.

Integral gain (Ki): Larger integral gain implies steady-state errors are eliminated faster. However, the trade-off is a larger overshoot

Derivative gain $(\mathrm{Kd})$ : Larger derivative gain decreases overshoot but slows down transient response and may lead to instability due to signal noise amplification in the differentiation of the error.

The Ziegler-Nichols tuning developed by John G. Ziegler and Nathaniel B. Nichols is performed by setting the $I$ (integral) and $D$ (derivative) gains to zero. The "P" (proportional) gain, $\mathrm{Kp}$ is increased from zero until it reaches the (ultimate gain) $\mathrm{Ku}$, at which the output of the control loop has stable and consistent oscillations. $\mathrm{Ku}$ and $\mathrm{Tu}$ (the oscillation period) are used to set the P, I, and D gains depending on the type of controller used. Ziegler-Nichols tuning creates a quarter wave decay. 


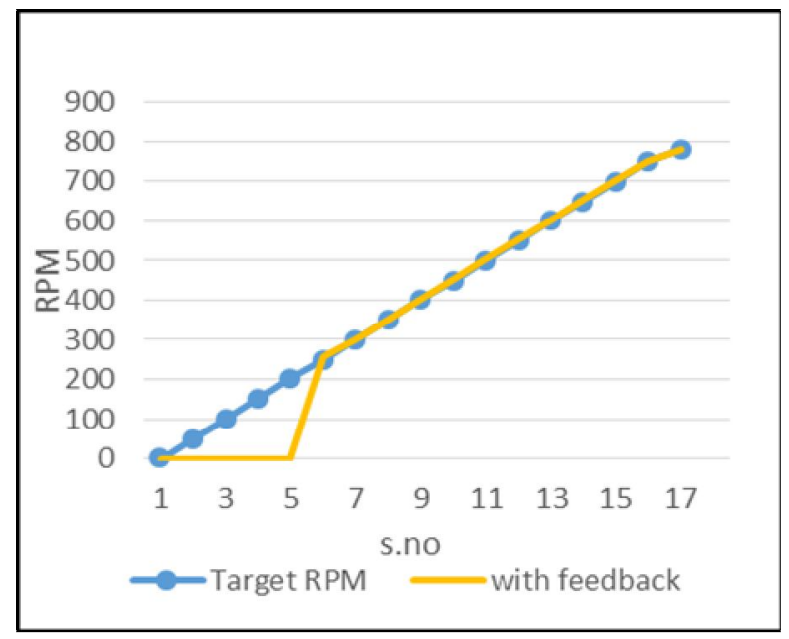

Figure 6. Motor response with feedback

\section{Load stabilization}

When a constant load is applied to the motor after a given settling time, the impact of the load will reduce the revolutions per minute.

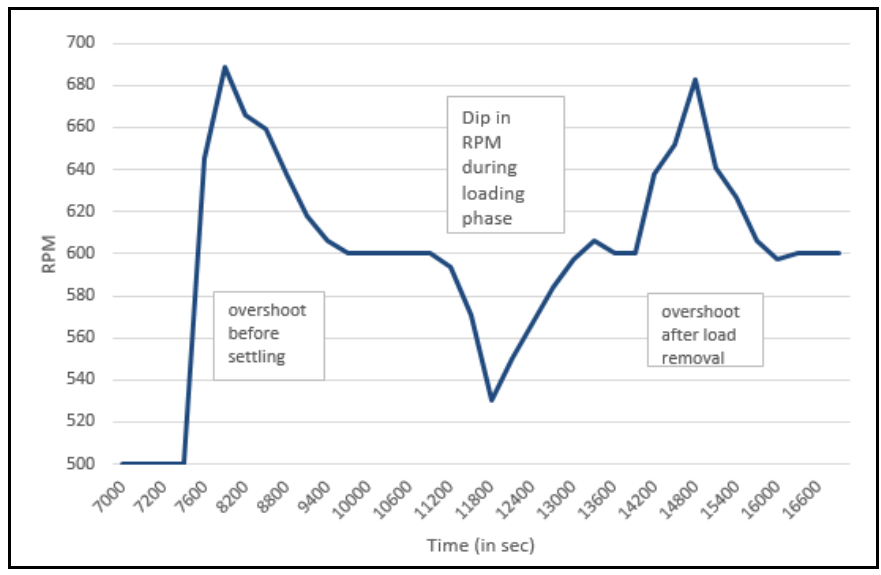

Figure 7. Load-Unload characteristics

The reduction in the RPM creates an error between the set and the actual point. This error results in increasing the PWM based on the algorithm to diminish the error and the set is achieved. When the load is removed, the actual point shoots up to a value greater than the set point. This error is corrected by the algorithm and the motor settles at the desired speed.

\section{IV.CONCLUSION}

The motor response is observed to be nonlinear and the PWM mapping using the direct proportional method doesn't make the motor run at the target RPM. This concludes that the motor doesn't have a linear behaviour in the whole operating range. The feedback system adjusts the PWM in a calculated amount with the help of a PID controller technique. Thus, the implementation of this algorithm was investigated successfully using this case study. 


\section{REFERENCES}

[1] Atul Kumar Dewangan, Nibbedita Chakraborty, Sashi Shukla, Vinod Yadu ,” PWM Based Automatic Closed Loop Speed Control of DC Motor", International Journal of Engineering Trends and Technology- Volume3Issue2- 2012

[2] Gopal K. Dubey, "Fundamentals of Electric Drives", Narosa Publishing House New Delhi, 1989

[3] Gopal P. Gawande, Shruti V. Gavhale, Irshad A. Zariye, Sagar P. Ritpurkar, "Review of Speed Control and Automatic Braking System", International Journal of Engineering Research \& Technology (IJERT) Vol. 3 Issue 2, February - 2014

[4] Md. Masud Rana, Md. Sahabuddin, Shourov Mondol, "Design and Implementation of a Digital Tachometer", International Journal of Scientific Engineering and Technology, ISSN:2277-1581, Volume No.5 Issue No.1, pp: 85-88

[5] Thirupathi Allam, Matla Raju, S.Sundeep Kumar," Design of PID controller for DC Motor Speed Control Using Arduino Microcontroller [6] Wayne H. Wolf, "Hardware-Software Co-Design of Embedded Systems", Proceedings of the IEEE, VOL. 82. NO. 7, JULY 1994

[7] PID Control: brief A introduction and a http://www.academia.edu/7729313/PID_Control_A_brief_introduction_and_guide_using_Arduino

[8] Ziegler-Nichols Method

http://kurser.iha.dk/m/mtpri1/control/3_PID/f_ziegler/ziegler_nichols_method.htm 